\title{
Inljnltgverzeidunis.
}

Einleitung . . . . . . . . . . XIX

\section{Bürgerlides Gejebbudy.}

Crites $B$ u西.

\section{Mllgemeitter Ieil.}

Erfter Mbjünitt. Berfonen

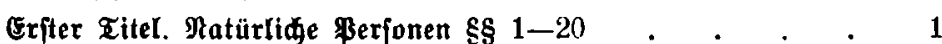

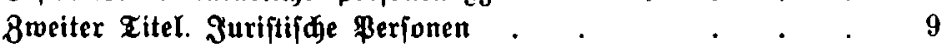

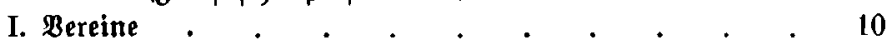

1. Âgemeine Borfdriften $\$ \S 21-54 \quad$. $\quad . \quad . \quad$. 10

2. Cingetragene Bereine $\$ \$ 55-79 \quad$. $\quad . \quad . \quad$. 22

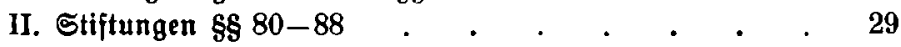

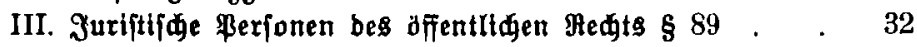

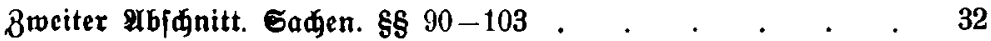

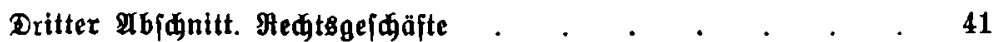

Erfter Zitel. GefdăftBfähigleit $\$ \$ 105-115$. . . . 42

Zweiter Fitel. Willenserflärung $\$ \S 116-144$. . . . $\quad$. 47

Dritter Titel. Bertrag $\$ \S 14 \overline{5}-157$. . . . . . . . . $\quad .64$

Bierter Ritel. Bebingung. Beitbeftimmung $\$ \S 158-163$. $\quad$. 69

Z̛ünfter Ditel. Bertretung. Bollmadyt $\$ 8$ 164-181 . . . 71

Sedifter \&itel. Einmilligung. Benehmigung $\$$ 182-185 . . 80

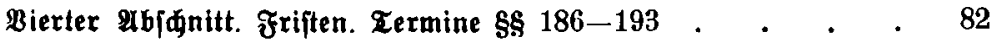

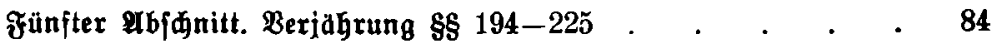










\section{Nrdht ber Sdjuldberhältuilie.}



Erfter Titel. Berpfriftung zur Beiftung $\$ \$ 241-292$. . 105

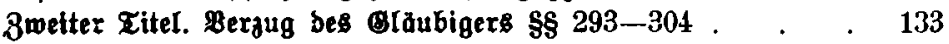

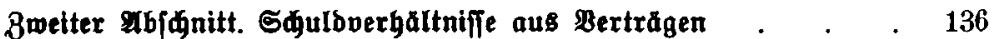

Erfter Ritel. Begrïndung, Jnhalt bes Bertrags \&8 305-319 . 136

Bweiter Zitel. Begenjeitiger Bertrag $\$ 8320-327$. . . 143

Dritter Titel. Beripreøen ber Beiftung an einen Dritten $\$ 8328-335$. $\quad . \quad . \quad . \quad . \quad . \quad . \quad . \quad 149$

Bierter Fitel. Draufgabe. Bertragsitrafe \$\$ 336-345 . . 153

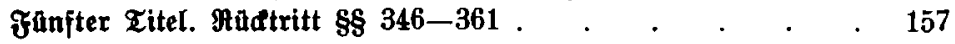

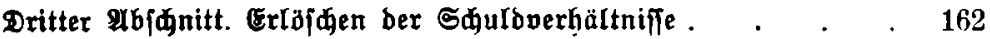

Erjter Titel. Erfüllung \$\$ $362-371$. . . . . . . 162

3weiter Ritel. Einterlegung $\$ \$ 372-386$. . . . . . . 167

Dritter Titel. Aufrednung \$8 387-396 . . . . . . 172

Bierter Ritel. Erlaß̉ $\$ 397 \quad$. . . . . . . . . . 176

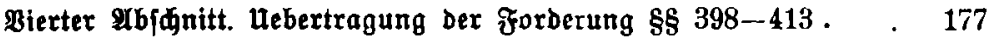

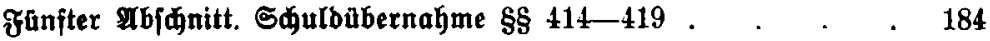

Sedjter $\mathfrak{A b j}$ jnitt. Mtebrheit von Sdulbnern und Bläubigern

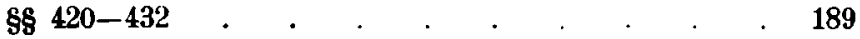

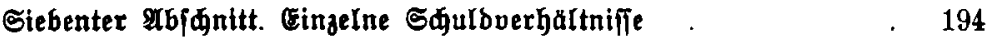



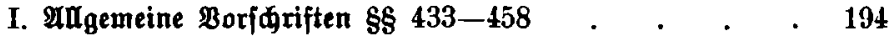

II. Bewåbrleiftung wegen Mångel ber Sadje $\$ 8459-493$. 205

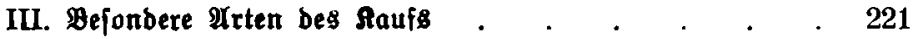

1. Rauf nad \$robe. Sauf auf \$robe $\$ 85494-496$. 221

2. Miebertauj $\$ 8497-503$. . . . . . . 222

3. Bortauf $\$ 804-514$. . . . . . . . 224

IV. Tauj内 \& 515 . . . 227

8 weiter Titel. Sめentung \$8 516-534 . $\quad 228$

Dritter Titel. Mhiete. \$ađt . . . . . . . 235

I. Miete $\$ 8535-580$. . . . . 235





Zünfter Titel. Darlehen $\$ \$ 607-610$. . . . . . . . 263

Sedfter Ritel. Dienftwertrag $\$ 8611-630$. . . . . . 265 
Gette

Siebenter Titel. Merfvertrag $\$ 831-651$. . . . . 275

IAter Titel. Mallerpertrag $\$ \$ 652-656$. . . . . . . 284



BeGnter Ritel. Muftrag $\$ 862-676$. . . . $\quad 290$

Elfter Titel. Befdaftsführung ohne $\mathfrak{A} u f t r a g$ \$ $\$ 877-687 \quad 296$

8 wàlftex Titel. Berwahrung $\$ \$ 688-700$. . . $\quad . \quad 300$

Dreigehntex Ritel. Einbringung von Saden bei Sajtwirten

\$s $701-704.6 . \quad . \quad . \quad . \quad . \quad . \quad .305$

Bierzebnter Ritel. Sejellídaft \&8 705-740 . . . . 307

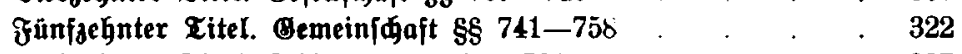

Sedałehnter Titel. Reibrente $\$ \S 759-761$. . . . 327

Siebzebntex Iitel. Spiel. Wette \$\$ 762-764 . . . 329

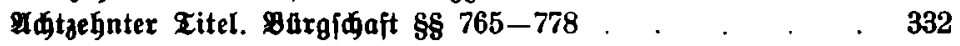

Reunzehnter Ritel. Bergleid $\$ 779 \quad$. $\quad . \quad$. $\quad . \quad . \quad$. 341

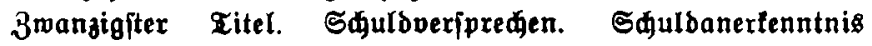

$\$ \S 780-782$. $\quad . \quad . \quad . \quad . \quad . \quad . \quad . \quad . \quad 342$

Einunbzwanaigiter Ritel. Anweifung 88 783-792 . . . 345

3weiundzmanjigiter Ritel. Sđjulbveridreibung auf ben Ingaber

$\$ \$ 793-808$.

Dreiundzwanjigiter Sitel. Borlegung von Sałen \$s $809-811 \quad 357$

Bierundjwanzigiter Eitel. Ungeredtfertigte Bereiderung $\$ 8812$ bts $822, . \quad . \quad . \quad . \quad . \quad . \quad . \quad . \quad 359$



\section{Drittes $\$$ úd.}

\section{Eadjenredft.}

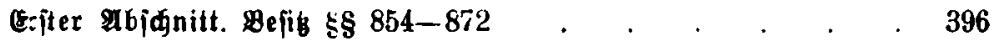


ftüden $\$ \S 873-902$. $5 . \quad . \quad . \quad . \quad . \quad . \quad$. 405

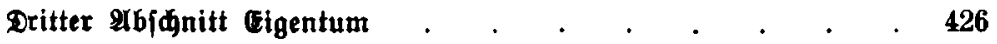

Erfier Eitel. Inhalt bes Eigentums \&s 903-924 . . . 426

Bweiter Titel. Erwerb und Berluft bes Eigentums an Grunb=



Dritter Ritel. Ermerb und Berluft bes Eigentums an bewegltden Sađen . . . . . . . . . . . $4 \mathbf{4 2}$

I. uebertragung $\$ \$ 929-93 i$. . . . . . . 442

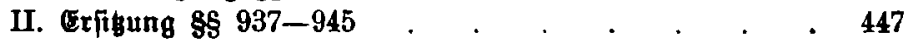


III. Berbindung. Bermifđung. Berarbeitung $\$ \$ 946-952 \quad 4 \quad 49$

IV. Ermexb von Erzeugnifen und ponitigen Beftandteilen $\$ 8953-957$. $\quad . \quad . \quad . \quad . \quad . \quad . \quad . \quad .4 \tilde{\mathbf{5} 2}$

V. Aneignung \$§ 958-964 . . . . . . . . . . . . $\quad 454$

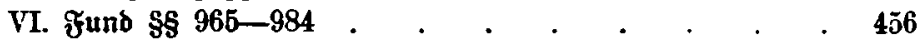

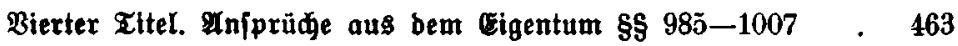

Fünfter Fitel. Miteigentum \$\$ 1008-1011 . . . . 473

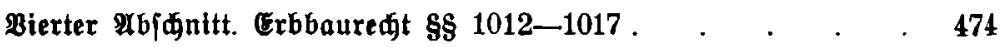

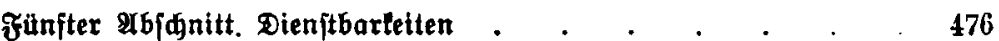

Eriter Ritel. \&rundoienjtbarteiten $\$ \S 1018-1029$. . . 476

3weiter Ritel. Nieß̧braud

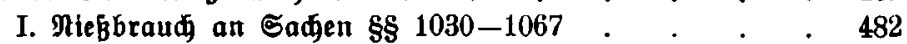

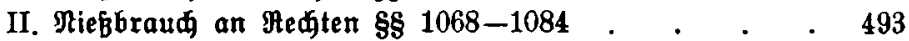

III. Nieß̧braud an etnem Bermögen §§ 1085-1089 . . 497

Dritter Titer. Befđräntte perfönlide Dienttbarteiten \$\$ 1090 bis 1093 . . . . . . . . . . . 499

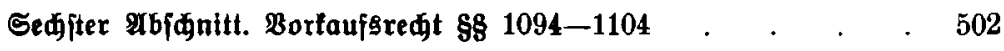

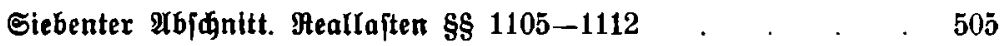

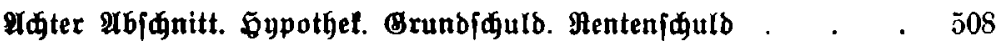

Eriter Zitel. Gypothet \$8 $1113-1190 \quad$. . . . . . 508

Bweiter Ritel. Grunbjould. Mentenjould . . . . . .

I. Orunbjaulo \$8 $1191-1198$. . . . . . . . 553

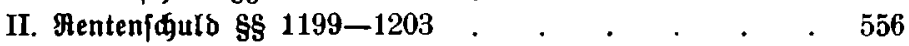

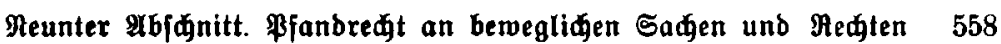

Erfter Titel. Bjandredt an bewegliden Sadjen \$8 1204-1272 558

Broeiter Ettel. \$fanbredt an Medten $\$ \$ 1273--1296$. . 580

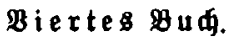

Familienredft.



Erfter Fitel. Berlöbnis \$8 1297-1302 . . . . . . . 589

3weiter Fitel. Eingehung ber Ehe \$s 1303-1322 . . . 592

Dritter ritel. Ntixtigteit und Ânfebtbarteit ber Ege 881323 bt8 1347 . . . . . . . . . . 600

Bterter Fitel. Wieberverheiratung im Falle ber Fobeserflärung \$\$ 1348-1352 . . . . . . . . . . . . 610 
Fünfter Ritel. Wirfungen ber EGe im allgemeinen :\$\$ 1353 b) 1362 . . . . . . . . . . 611 Sedfter Ritel. Egelide Büterredt . . . . . . $\quad 617$ I. Sejeglides Büterredt . . . . . . . 618

1. $\mathfrak{A}$ gemeine Boridriften \$8 1363-1372 . $\quad$. $\quad 618$

2. Berwaltung unb Nuønießung \$8 $1373-1409$. . 621

3. Squldenfaftung $\$ 81410-1417 \quad$. $\quad . \quad$. 635

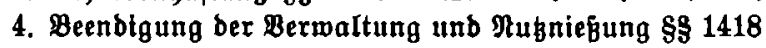



5. Buttertrennung $\$ \S 1426-1431$. $\quad . \quad . \quad . \quad .642$

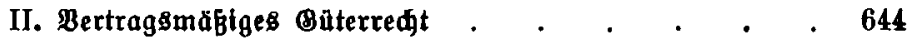

1. All gemetne Borjøriften \$8 1432-1436 . . . 644

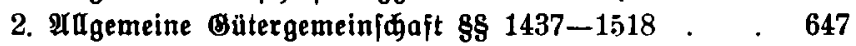

3. Errungenf西aftsemeinifaft \$§ $1519-1548$. $\quad 683$

4. Fałrnisgemeinjđaft $\$ 81549-1557$. . . . . 691

III. Baterredtstegtfter \$\$ $1558-1563$. . . . . 694

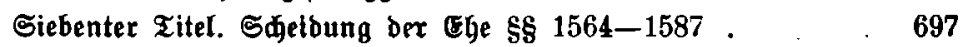



3weiter 2lbfdnitt. Bermandifafaf . . . . . . . 714

Eriter Ritel. AXgemeine Porjøriften $\$ \$ 1589,1590 . \quad 714$

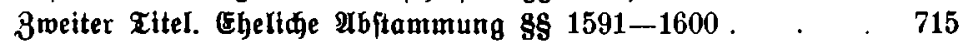

Dritter Titel. unterhaltopflidgt \$8 $1601-1615$. . . . 719

Bierter Sitel. Medtltide Stellung ber eheliden Sinber . $\quad 728$

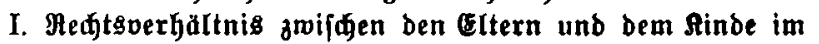
allgemeinen $\$ \$ 1616-1625$. . . . . . . 728

II. Clterlide Bewalt $\$ 1626 . \quad . \quad . \quad . \quad . \quad . \quad 733$

1. Elterlifie Bemalt des Bater $\$ 8$ 1627-1683 . . 734

2. EIterlitie Bewalt ber Mtutter \&8 1684-1698 . . 763

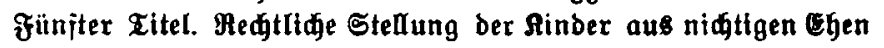
$\S 81699-1704$. . . . . . . . : . . . 769

Sedjter Ritel. Medtride Stellung ber unebeliden Ainder \$§ 1705 bis 1718 . . . . . . . . . . 772

Siebenter Ritel. Regitimation unebeliäer Sinder . . . 781

I. Begitimation burd nadjolgende Ehe \$8 1791-1722 . 781

II. Cgelidłeitzerflărung $\$ \$ 1723-1740$. . . . 783

U4ter Titel. Unnałme an findes Statt \$S 1741-1772 • 789

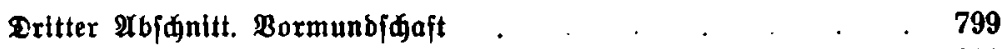

Erfter Titel. Bormundjøaft über Minderjährige . . . 801

I. Inorbnung ber Bornundføaft \$\$ 1773-1792 . . 801

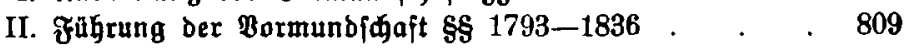




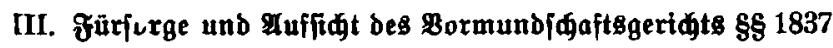
bis 1848

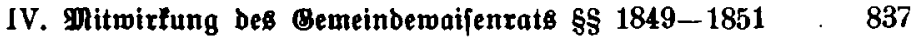

V. Befreite Bormund/两aft \$8 1852-1857 . . . , 838

VI. Familienrat \$s $1858-1881$. $\quad . \quad . \quad . \quad . \quad . \quad 840$

VII. Beenbigung ber Bormunbfdajt \$\$ 1882-1895 $\quad . \quad 847$

8weiter Ritel. Bormundjøaft über Bolladgrige $\$ 8: 1896-1908 \quad 852$

Dritter \&itel. Bflegífajt \$\& 1909-1921 . . . . . 85̃6

Fũ ñ ties $\mathfrak{B}$ u d.

\section{(5tbredt.}

Eriter abjđjnitt. Erbjulge $\$ \$ 1922-1941$. . . . 863

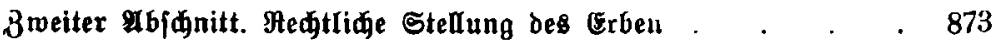

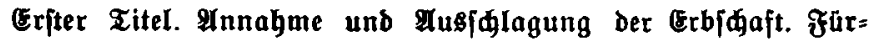

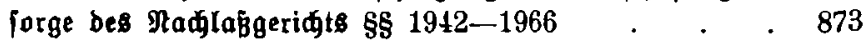

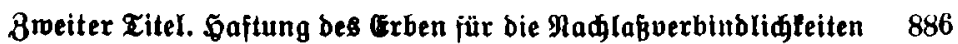

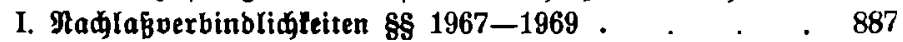

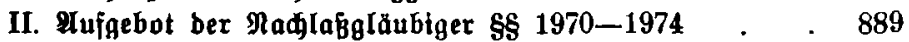

III. Befđrănfung ber Đaftung Deß Eerben \$8 1975-1992 . 892

IV. Inventarerridtung. Unbejđräntte Sajtung bes Erben $\$ 81993-2013$. . . . . . . . . 903

V. Yuffdiebende Cinreben $\$ \S 2014-2017$. . . 912

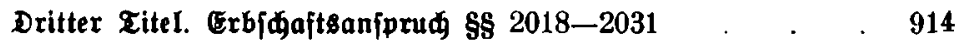

Bierter 足tel.jptehrbeit bex Krben . . . . . . 920

I. Neqtserbăltnis ber Crben untereinander $\$ \$ 2032-2057 \quad 920$

II. Aedtovergattnis zwlfdien ben Erhen und ben Madala $\$=$ glăubigern \$§ 2058-2063 $\quad . \quad . \quad . \quad . \quad . \quad . \quad .933$

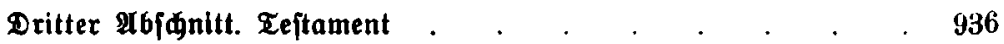

Erftex Tltel. Â巛

3weiter Sitel. Erbeinjegung 88 2087-2099 . . . . 945

Dritter Ritel. Einjebung eines Nađarben \$s 2100-2146 . 949

Bierter Ritel. Bermädtni\$ 83 2147-2191. . . . 966

Fünfter Ritel. Puflage 88 2192-2196 . . . . . $\quad 980$

Sediter Titel. Teftamentspollfteder 88 2197-2228 . . . 982

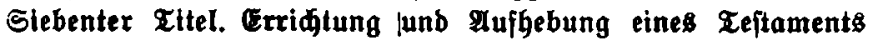

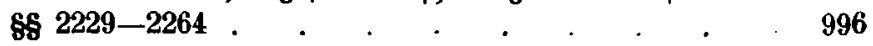

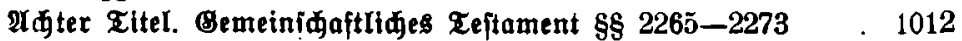




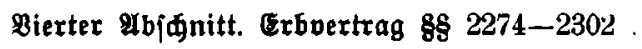

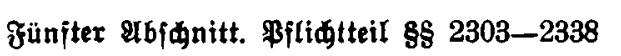



Siebenter 2 (1)

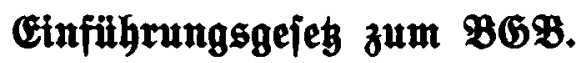

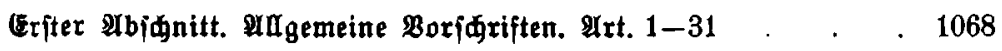






Bandesgejęen. Art. 55-152 . . . . . . 1107

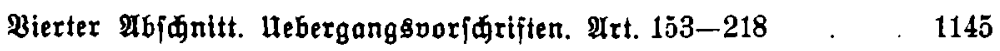

Alphabetifdes Regiftei . . . . . . . . . . 1179 